\title{
Increasing the surface density of adsorbed hydrogen with coordinatively unsaturated metal centers in metal-organic frameworks
}

\author{
Yun Liu ${ }^{\dagger,}$, Houria Kabbour ${ }^{\S}$, Craig M. Brown ${ }^{\dagger}$, Dan A. Neumann ${ }^{\dagger}$, Channing C. Ahn ${ }^{\S, *}$ \\ $\dagger$ NIST Center for Neutron Research, 100 Bureau Drive, Gaithersburg, MD, USA \\ $\$$ Department of Materials Science and Engineering, University of Maryland, College \\ Park, MD, USA \\ $\S$ Division of Engineering and Applied Science, California Institute of Technology, \\ Pasadena, CA, USA
}

* To whom correspondence should be addressed. E-mail: cca@caltech.edu

\section{Supporting Information}

Materials and Methods

\section{Tables S1-S10}

Figs. S1-S4 


\section{BET analysis}

BET and Langmuir surface areas were analyzed by Micromeritics Analytical Services (Norcross, Georgia) ${ }^{1}$, where the skeletal density was also measured. The samples were handled in inert atmosphere and treated similarly as for our Sieverts measurements (120 ${ }^{\circ} \mathrm{C}$ under vacuum prior to measurements).

\section{Neutron powder diffraction experiments}

Neutron powder diffraction data were collected on the High Resolution Neutron Powder Diffractometer BT-1 at the NIST Center for Neutron Research (NCNR) with a $\mathrm{Ge}(311)$ monochromator and using in-pile collimation of 15 minutes of arc, corresponding to a wavelength of $2.0787 \AA$. Measurements were taken as a function of Deuterium loading at a temperature of $3.5 \mathrm{~K}$ with measurement times of around 9 hours.

All sample transfers were performed in a helium-filled glove box equipped with water and oxygen monitors. The sample was evacuated using a turbo pump $\left(10^{-3} \mathrm{~Pa}\right)$ while heating to $120{ }^{\circ} \mathrm{C}$. After degassing at $120{ }^{\circ} \mathrm{C}$ for about 12 hours, the sample was cooled and transferred to a cylindrical vanadium can equipped with a capillary gas line and a packless valve, and sealed with an indium O-ring. The sample was mounted onto a sample stick, equipped with a stainless-steel gas line with an additional valve for a top loading closed-cycle helium refrigerator (CCR). $1.412 \mathrm{~g}$ of MOF-74 was used for the neutron scattering measurements.

During the experiments, a known amount of hydrogen (deuterium) gas was loaded into the sample, which was maintained at a temperature of $77 \mathrm{~K}$ until no pressure drop can be recorded for at least 1 minute. The sample was then cooled down to the base temperature of the CCR $(4 \mathrm{~K})$ over one hour in order to perform measurements. In all cases the outgas pressure reading was zero well before reaching $25 \mathrm{~K}$ except for the loadings at $4.2 \mathrm{D}_{2}: \mathrm{Zn}$.

\footnotetext{
${ }^{1}$ Certain commercial equipment, instruments, or materials are identified in this document. Such identification does not imply recommendation or endorsement by the National Institute of Standards and Technology nor does it imply that the products identified are necessarily the best available for the purpose.
} 
Neutron scattering diffraction patterns were analyzed using the Rietveld refinement method. ${ }^{2}$ The program EXPGUI was used to perform all refinements. ${ }^{3}$ The model of the bare material was refined first and it was used as the starting point for subsequent refinements of the $\mathrm{D}_{2}$-loaded samples. Deuterium molecules are treated as point scatters with double occupancy since they are expected to be quantum mechanically spherically averaged in the ground state. One soft-constraint was imposed to require the benzene $\mathrm{C}-\mathrm{H}$ bond distance to be around $1.1 \AA$. The coordinates and Debye-Waller factors of all other atoms were allowed to vary. An anisotropic thermal factor was used for the $\mathrm{D}_{2}(1)$ site since it always resulted in a better $\chi^{2}$ of the refinements. All other $\mathrm{D}_{2}$ adsorption sites used isotropic thermal factors.

\section{Inelastic neutron scattering experiments with para-hydrogen}

The same sample used in the neutron powder diffraction experiments was directly transferred to a long-tail top loading CCR for inelastic neutron scattering (INS) experiments. The INS spectra were collected using the Filter Analyzer Neutron Spectrometer (FANS) $^{4}$ at the NCNR, which is designed for neutron vibrational spectroscopy. The energy resolution is between $1.2 \mathrm{meV}$ to $2.0 \mathrm{meV}$ over the energy transfer ranges studied.

Hydrogen gas was used during the measurements to take advantage of its large incoherent neutron scattering cross section. $\mathrm{A}_{2}$ molecule is a very good quantum rotor due to its light mass and consists of two indistinguishable fermions (protons) that require the wave function to be anti-symmetric. If the nuclear spins of two protons are antiparallel, $\mathrm{H}_{2}$ is said to be in a para state, otherwise, it is in an ortho state. The quantum rotation number, $J$, of a $\mathrm{H}_{2}$ molecule thus has to be even for a para- $\mathrm{H}_{2}$ and odd for an ortho- $\mathrm{H}_{2}$. At room temperature, only one fourth of $\mathrm{H}_{2}$ molecules are in the para state. Usually, the conversion rate between states is very slow. We used para- $\mathrm{H}_{2}$ (created from hydrogen flow through an off-line paramagnetic catalyst) for the INS experiments.

\footnotetext{
${ }^{2}$ H. M Rietveld, Acta. Cryst. 22, 151 (1967).

${ }^{3}$ B. H. Toby, J. Appl. Cryst. 34, 210 (2001).

${ }^{4}$ T. J. Udovic, D. A. Neumann, J. Leao, C. M. Brown, Instrum. Methods A517, 189 (2004).
} 
A neutron scattered by a $\mathrm{H}_{2}$ molecule can induce the required nuclear spin flip to convert a para/ortho $\mathrm{H}_{2}$ to an ortho/para $\mathrm{H}_{2}$. This para-ortho or ortho-para transition is associated with the change of the rotational quantum number, $J$, from even/odd to odd/even, and has a large neutron scattering cross-section that is proportional to the incoherent neutron scattering cross-section of the proton. INS spectra of the adsorbed $\mathrm{H}_{2}$ were obtained by subtracting the INS spectrum of the bare materials. Since we use para$\mathrm{H}_{2}$, the excitations involving the para-ortho transition dominates the spectra. For a free hydrogen molecule, the para-ortho transition is usually associated with the $J=0$ to $J=1$ excitation occurring with an energy of $14.7 \mathrm{meV}$, which can be directly measured by INS but not optical spectroscopies. The local potential of a host material will generate rotational barriers for the adsorbed $\mathrm{H}_{2}$ which may cause the $J=1$ state to split into its three sublevels. Additionally, the translation excitations of the $\mathrm{H}_{2}$ molecule may be coupled to the rotational transition and complicate the observed spectra. Hence, the INS spectra of adsorbed $\mathrm{H}_{2}$ may show complex features with multiple peaks in the spectrum containing rich information about the host material and the hydrogen interactions.

During the INS experiments, para- $\mathrm{H}_{2}$ gas was loaded at about $50 \mathrm{~K}$ and slowly cooled down to $4 \mathrm{~K}$ for measurements. The pressure gauge read zero well before the temperature reached $25 \mathrm{~K}$. Fig. S2 shows the INS spectra of $\mathrm{H}_{2}$ at $0.8 \mathrm{H}_{2}: \mathrm{Zn}$ and 1.6 $\mathrm{H}_{2}: \mathrm{Zn}$ loadings. From our understanding of the structural work in this paper, all the peaks shown in Fig. S2 for $0.8 \mathrm{H}_{2}: \mathrm{Zn}$ must be due to the motions of $\mathrm{H}_{2}$ adsorbed at the $\mathrm{Zn}^{2+}$ site. The detailed analysis of the origins of different peaks is still underway and will be presented in the future. The sharp peaks at $8.3 \mathrm{meV}$ and $11.3 \mathrm{meV}$ are due to the rotational transition associated with a para-ortho transition. The peaks from $18 \mathrm{meV}$ to $25 \mathrm{meV}$ may be due to either rotational transition or the coupling between translational and rotational transition. Since we can be sure that all peaks originate from the excitations of $\mathrm{H}_{2}$ at one site it, is evident that one needs to be very careful when assigning peaks at differing frequencies to $\mathrm{H}_{2}$ adsorbed at different adsorption sites. In the 1.6 $\mathrm{H}_{2}: \mathrm{Zn}$ case, $\mathrm{H}_{2}$ populates the second adsorption site. The spectrum shows that the peak at $11 \mathrm{meV}$ becomes broader, potentially due to the appearance of a new peak close to 11 $\mathrm{meV}$, associated with the second adsorption site. New peaks have also appeared at around 
14 and $16 \mathrm{meV}$ and can be regarded as a characteristic feature of adsorbed $\mathrm{H}_{2}$ at the second adsorption site.

\section{Temperature dependent INS spectra of $\mathrm{H}_{2}$ in MOF-74}

In order to evaluate the relative binding strength of the adsorbed $\mathrm{H}_{2}$ at the first three adsorption sites, we have performed temperature dependent experiments. After loading $2.8 \mathrm{H}_{2}: \mathrm{Zn}$ and slowly cooling the sample to $4 \mathrm{~K}$, the sample was warmed up to a degassing temperature, $T_{p}$, where the sample was degassed for approximately 30 mins. Re-cooling the sample to $4 \mathrm{~K}$ to perform the measurements allows us to neglect possible differences in intensities due to variations of the Debye-Waller factors. These procedures were repeated for different $T_{p}$. The degas temperatures were $30 \mathrm{~K}, 50 \mathrm{~K}$, and $70 \mathrm{~K}$. The resulting INS spectra are shown in Fig. S3. When $T_{p}=30 \mathrm{~K}$, there are some peaks between $14 \mathrm{meV}$ to $16 \mathrm{meV}$ indicating that more than one adsorption site was occupied. When $T_{p}=50 \mathrm{~K}$, the total intensity of the spectrum is comparable to that of the spectrum of $0.8 \mathrm{H}_{2}: \mathrm{Zn}$. Therefore, there is only one site occupied by $\mathrm{H}_{2}$ after degassing the sample at $50 \mathrm{~K}$ and cooling to $4 \mathrm{~K}$. Degassing the sample at $70 \mathrm{~K}$ could not remove all of the hydrogen molecules as indicated by the remaining scattering intensity of the spectrum. Hence, the binding strength of the first site should be significantly larger than the second and third binding sites while the binding strength difference between the $2^{\text {nd }}$ and $3^{\text {rd }}$ sites is much smaller. 
Table S1. Refinement details and $\mathrm{D}_{2}$ loadings at individual adsorption sites obtained from powder neutron diffraction. Estimated standard deviations from the Rietveld analysis are given in parentheses.

\begin{tabular}{|c|c|c|c|c|c|c|}
\hline \multirow{2}{*}{$\mathrm{D}_{2}$ loading $\left(\mathrm{D}_{2}: \mathrm{Zn}\right)$} & \multicolumn{5}{|c|}{ Refined $\mathrm{D}_{2}$ Occupancies } & Rietveld \\
\cline { 2 - 6 } & Site 1 & Site 2 & Site 3 & Site 4 & Total & $\chi^{2}$ \\
\hline 0 & N/A & N/A & N/A & N/A & N/A & 1.051 \\
$1 / 3$ & $0.38(1)$ & & & & $0.38(1)$ & 1.665 \\
$2 / 3$ & $0.73(1)$ & & & & $0.73(1)$ & 1.166 \\
1.0 & $0.94(2)$ & & & & $0.94(2)$ & 1.182 \\
2.0 & $1.03(2)$ & $1.02(3)$ & $0.12(2)$ & & $2.19(4)$ & 1.092 \\
3.0 & $0.88(2)$ & $0.90(3)$ & $0.89(2)$ & $0.09(1)$ & $2.76(4)$ & 1.061 \\
$4.2^{\mathrm{a}}$ & $0.88(1)$ & $1.06(2)$ & $1.04(1)$ & $1.00(1)$ & $3.98(3)^{\mathrm{a}}$ & 1.491 \\
\hline
\end{tabular}

a) We purposefully load more $\mathrm{D}_{2}$ gas to saturate all the adsorption sites in MOF-74. There was a small residual gas pressure at $16.5 \mathrm{~K}$. Since we did not observe any diffraction peaks from solid $\mathrm{D}_{2}$, any excess $\mathrm{D}_{2}$ molecules $\left(\approx 0.2 \mathrm{D}_{2}: \mathrm{Zn}\right)$ may be adsorbed elsewhere in the system or adsorbed on the surface of the particles in a disordered or liquid-like manner. 
Table S2. Rietveld refinement results for the bare MOF-74. $a=b=25.887(3) \AA, c=$ 6.816(1) A. Estimated standard deviations from the Rietveld analysis are given in parentheses.

\begin{tabular}{|c|c|c|c|c|c|c|}
\hline & $\mathrm{x}$ & $\mathrm{y}$ & $\mathrm{z}$ & Multiplicity & Occupancy & $\mathrm{U}_{\text {iso }}\left(\AA^{2}\right)$ \\
\hline $\mathrm{Zn}$ & $0.384(2)$ & $0.348(2)$ & $0.151(5)$ & 18 & 1.0 & $0.15(1)$ \\
$\mathrm{O} 1$ & $0.324(2)$ & $0.291(2)$ & $0.364(8)$ & 18 & 1.0 & $0.20(2)$ \\
$\mathrm{O} 2$ & $0.298(1)$ & $0.229(1)$ & $0.611(4)$ & 18 & 1.0 & $0.073(9)$ \\
$\mathrm{O} 3$ & $0.355(1)$ & $0.280(1)$ & $0.004(4)$ & 18 & 1.0 & $0.065(8)$ \\
$\mathrm{C} 1$ & $0.323(2)$ & $0.245(2)$ & $0.416(7)$ & 18 & 1.0 & $0.28(3)$ \\
$\mathrm{C} 2$ & $0.330(2)$ & $0.204(1)$ & $0.291(5)$ & 18 & 1.0 & $0.15(1)$ \\
$\mathrm{C} 3$ & $0.3451(7)$ & $0.2247(8)$ & $0.091(2)$ & 18 & 1.0 & $0.012(4)$ \\
$\mathrm{C} 4$ & $0.3533(8)$ & $0.1845(9)$ & $-0.039(2)$ & 18 & 1.0 & $0.031(5)$ \\
$\mathrm{H}$ & $0.364(1)$ & $0.193(1)$ & $-0.142(4)$ & 18 & 1.0 & $0.003(9)$ \\
\hline
\end{tabular}


Table S3. Rietveld refinement results for $1 / 3 \mathrm{D}_{2}: \mathrm{Zn} . a=b=25.873(3) \AA, c=6.835(1) \AA$.

Estimated standard deviations from the Rietveld analysis are given in parentheses.

\begin{tabular}{|c|c|c|c|c|c|c|}
\hline & $\mathrm{x}$ & $\mathrm{y}$ & $\mathrm{z}$ & Multiplicity & Occupancy & $\mathrm{U}_{\text {iso }}\left(\AA^{2}\right)$ \\
\hline $\mathrm{Zn}$ & $0.385(2)$ & $0.362(2)$ & $0.146(5)$ & 18 & 1.0 & $0.13(1)$ \\
$\mathrm{O} 1$ & $0.320(2)$ & $0.294(2)$ & $0.338(6)$ & 18 & 1.0 & $0.15(1)$ \\
$\mathrm{O} 2$ & $0.298(1)$ & $0.228(1)$ & $0.611(4)$ & 18 & 1.0 & $0.073(9)$ \\
$\mathrm{O} 3$ & $0.361(1)$ & $0.283(1)$ & $0.028(4)$ & 18 & 1.0 & $0.089(9)$ \\
$\mathrm{C} 1$ & $0.321(2)$ & $0.245(1)$ & $0.431(5)$ & 18 & 1.0 & $0.15(1)$ \\
$\mathrm{C} 2$ & $0.325(1)$ & $0.204(1)$ & $0.290(4)$ & 18 & 1.0 & $0.12(1)$ \\
$\mathrm{C} 3$ & $0.3427(9)$ & $0.223(1)$ & $0.089(2)$ & 18 & 1.0 & $0.035(6)$ \\
$\mathrm{C} 4$ & $0.3531(7)$ & $0.1854(7)$ & $-0.033(2)$ & 18 & 1.0 & $0.01(4)$ \\
$\mathrm{H}$ & $0.363(1)$ & $0.194(1)$ & $-0.137(3)$ & 18 & 1.0 & $0.01(8)$ \\
$\mathrm{D}_{2}(1)$ & $0.2003(8)$ & $-0.010(2)$ & $0.023(4)$ & 18 & $0.77(3)$ & $\mathrm{a}$ \\
\hline
\end{tabular}

a) The anisotropic thermal factor for $D_{2}(1)$ :

\begin{tabular}{|c|c|c|c|c|c|}
\hline $\mathrm{U}_{11}$ & $\mathrm{U}_{22}$ & $\mathrm{U}_{33}$ & $\mathrm{U}_{12}$ & $\mathrm{U}_{13}$ & $\mathrm{U}_{23}$ \\
$-0.02(1)$ & $0.23(4)$ & $0.17(3)$ & $0.07(2)$ & $-0.04(1)$ & $0.12(2)$ \\
\hline
\end{tabular}


Table S4. Rietveld refinement results for $2 / 3 \mathrm{D}_{2}: \mathrm{Zn} . a=b=25.857(2) \AA, c=6.847(1) \AA$.

Estimated standard deviations from the Rietveld analysis are given in parentheses.

\begin{tabular}{|c|c|c|c|c|c|c|}
\hline & $\mathrm{x}$ & $\mathrm{y}$ & $\mathrm{z}$ & Multiplicity & Occupancy & $\mathrm{U}_{\text {iso }}\left(\AA^{2}\right)$ \\
\hline $\mathrm{Zn}$ & $0.385(2)$ & $0.356(2)$ & $0.156(4)$ & 18 & 1.0 & $0.13(1)$ \\
$\mathrm{O} 1$ & $0.321(1)$ & $0.292(1)$ & $0.342(5)$ & 18 & 1.0 & $0.14(1)$ \\
$\mathrm{O} 2$ & $0.300(1)$ & $0.228(1)$ & $0.605(4)$ & 18 & 1.0 & $0.12(1)$ \\
$\mathrm{O} 3$ & $0.357(1)$ & $0.280(1)$ & $-0.005(4)$ & 18 & 1.0 & $0.071(8)$ \\
$\mathrm{C} 1$ & $0.319(2)$ & $0.244(1)$ & $0.433(5)$ & 18 & 1.0 & $0.19(2)$ \\
$\mathrm{C} 2$ & $0.329(1)$ & $0.207(1)$ & $0.298(3)$ & 18 & 1.0 & $0.096(9)$ \\
$\mathrm{C} 3$ & $0.3437(7)$ & $0.2249(9)$ & $0.092(2)$ & 18 & 1.0 & $0.026(6)$ \\
$\mathrm{C} 4$ & $0.3525(6)$ & $0.1854(6)$ & $-0.029(2)$ & 18 & 1.0 & $0.005(4)$ \\
$\mathrm{H}$ & $0.363(1)$ & $0.193(1)$ & $-0.146(3)$ & 18 & 1.0 & $0.012(8)$ \\
$\mathrm{D}_{2}(1)$ & $0.2003(7)$ & $-0.009(1)$ & $0.022(3)$ & 18 & $1.45(3)$ & $\mathrm{a}$ \\
\hline
\end{tabular}

a) The anisotropic thermal factor for $D_{2}(1)$ :

\begin{tabular}{|c|c|c|c|c|c|}
\hline $\mathrm{U}_{11}$ & $\mathrm{U}_{22}$ & $\mathrm{U}_{33}$ & $\mathrm{U}_{12}$ & $\mathrm{U}_{13}$ & $\mathrm{U}_{23}$ \\
$0.08(2)$ & $0.29(2)$ & $0.21(2)$ & $0.11(1)$ & $0.002(15)$ & $0.08(2)$ \\
\hline
\end{tabular}


Table S5. Rietveld refinement results for $1.0 \mathrm{D}_{2}: \mathrm{Zn} . a=b=25.854(2) \AA, c=6.851(1) \AA$.

Estimated standard deviations from the Rietveld analysis are given in parentheses.

\begin{tabular}{|c|c|c|c|c|c|c|}
\hline & $\mathrm{x}$ & $\mathrm{y}$ & $\mathrm{z}$ & Multiplicity & Occupancy & $\mathrm{U}_{\text {iso }}\left(\AA^{2}\right)$ \\
\hline $\mathrm{Zn}$ & $0.385(2)$ & $0.355(2)$ & $0.141(4)$ & 18 & 1.0 & $0.14(1)$ \\
$\mathrm{O} 1$ & $0.316(1)$ & $0.288(1)$ & $0.359(5)$ & 18 & 1.0 & $0.13(1)$ \\
$\mathrm{O} 2$ & $0.301(1)$ & $0.2291(9)$ & $0.609(3)$ & 18 & 1.0 & $0.059(8)$ \\
$\mathrm{O} 3$ & $0.356(1)$ & $0.279(1)$ & $0.003(3)$ & 18 & 1.0 & $0.054(7)$ \\
$\mathrm{C} 1$ & $0.323(2)$ & $0.244(1)$ & $0.434(5)$ & 18 & 1.0 & $0.21(2)$ \\
$\mathrm{C} 2$ & $0.329(1)$ & $0.209(1)$ & $0.293(3)$ & 18 & 1.0 & $0.11(1)$ \\
$\mathrm{C} 3$ & $0.3443(7)$ & $0.2251(8)$ & $0.091(2)$ & 18 & 1.0 & $0.017(5)$ \\
$\mathrm{C} 4$ & $0.3531(6)$ & $0.1855(7)$ & $-0.031(2)$ & 18 & 1.0 & $0.010(5)$ \\
$\mathrm{H}$ & $0.3663(9)$ & $0.193(1)$ & $-0.146(3)$ & 18 & 1.0 & $0.002(8)$ \\
$\mathrm{D}_{2}(1)$ & $0.1989(6)$ & $-0.0096(9)$ & $0.019(3)$ & 18 & $1.87(4)$ & $\mathrm{a}$ \\
\hline
\end{tabular}

a) The anisotropic thermal factor for $D_{2}(1)$ :

\begin{tabular}{|c|c|c|c|c|c|}
\hline $\mathrm{U}_{11}$ & $\mathrm{U}_{22}$ & $\mathrm{U}_{33}$ & $\mathrm{U}_{12}$ & $\mathrm{U}_{13}$ & $\mathrm{U}_{23}$ \\
$0.08(1)$ & $0.32(2)$ & $0.29(2)$ & $0.11(2)$ & $0.005(14)$ & $0.12(2)$ \\
\hline
\end{tabular}


Table S6. Rietveld refinement results for $2.0 \mathrm{D}_{2}: \mathrm{Zn} . a=b=25.851(2) \AA, c=6.862(1) \AA$.

Estimated standard deviations from the Rietveld analysis are given in parentheses.

\begin{tabular}{|c|c|c|c|c|c|c|}
\hline & $\mathrm{x}$ & $\mathrm{y}$ & $\mathrm{z}$ & Multiplicity & Occupancy & $\mathrm{U}_{\text {iso }}\left(\AA^{2}\right)$ \\
\hline $\mathrm{Zn}$ & $0.383(2)$ & $0.351(2)$ & $0.134(4)$ & 18 & 1.0 & $0.16(1)$ \\
$\mathrm{O} 1$ & $0.3179(8)$ & $0.2951(9)$ & $0.358(3)$ & 18 & 1.0 & $0.044(6)$ \\
$\mathrm{O} 2$ & $0.3047(9)$ & $0.2342(9)$ & $0.617(3)$ & 18 & 1.0 & $0.065(8)$ \\
$\mathrm{O} 3$ & $0.3562(9)$ & $0.2782(9)$ & $0.019(3)$ & 18 & 1.0 & $0.071(8)$ \\
$\mathrm{C} 1$ & $0.3192(6)$ & $0.2464(7)$ & $0.426(2)$ & 18 & 1.0 & $0.015(5)$ \\
$\mathrm{C} 2$ & $0.321(1)$ & $0.202(1)$ & $0.268(5)$ & 18 & 1.0 & $0.14(1)$ \\
$\mathrm{C} 3$ & $0.3430(6)$ & $0.2244(7)$ & $0.101(2)$ & 18 & 1.0 & $0.011(4)$ \\
$\mathrm{C} 4$ & $0.3511(7)$ & $0.1862(8)$ & $-0.035(2)$ & 18 & 1.0 & $0.029(6)$ \\
$\mathrm{H}$ & $0.366(1)$ & $0.194(1)$ & $-0.157(4)$ & 18 & 1.0 & $0.009(8)$ \\
$\mathrm{D}_{2}(1)$ & $0.1982(8)$ & $-0.009(1)$ & $0.056(2)$ & 18 & $2.05(5)$ & $\mathrm{a}$ \\
$\mathrm{D}_{2}(2)$ & $0.498(1)$ & $0.1312(9)$ & $-0.036(4)$ & 18 & $2.04(8)$ & $0.38(2)$ \\
$\mathrm{D}_{2}(3)$ & $0.740(6)$ & $0.215(6)$ & $0.10(3)$ & 18 & $0.24(6)$ & 0.24397 \\
\hline
\end{tabular}

a) The anisotropic thermal factor for $D_{2}(1)$ :

\begin{tabular}{|c|c|c|c|c|c|}
\hline $\mathrm{U}_{11}$ & $\mathrm{U}_{22}$ & $\mathrm{U}_{33}$ & $\mathrm{U}_{12}$ & $\mathrm{U}_{13}$ & $\mathrm{U}_{23}$ \\
$0.22(2)$ & $0.42(2)$ & $0.12(1)$ & $0.25(2)$ & $0.03(1)$ & $0.0005(156)$ \\
\hline
\end{tabular}


Table S7. Rietveld refinement for 3.0 $\mathrm{D}_{2}: \mathrm{Zn} . a=b=25.857(2) \AA ̊ ., c=6.869(1) \AA$.

Estimated standard deviations from the Rietveld analysis are given in parentheses.

\begin{tabular}{|c|c|c|c|c|c|c|}
\hline & $\mathrm{x}$ & $\mathrm{y}$ & $\mathrm{z}$ & Multiplicity & Occupancy & $\mathrm{U}_{\text {iso }}\left(\AA^{2}\right)$ \\
\hline $\mathrm{Zn}$ & $0.3737(9)$ & $0.344(1)$ & $0.146(3)$ & 18 & 1.0 & $0.049(8)$ \\
$\mathrm{O} 1$ & $0.3279(9)$ & $0.2962(9)$ & $0.347(4)$ & 18 & 1.0 & $0.049(8)$ \\
$\mathrm{O} 2$ & $0.2961(8)$ & $0.2257(8)$ & $0.597(3)$ & 18 & 1.0 & $0.014(5)$ \\
$\mathrm{O} 3$ & $0.3507(9)$ & $0.2787(8)$ & $0.015(3)$ & 18 & 1.0 & $0.011(5)$ \\
$\mathrm{C} 1$ & $0.3118(8)$ & $0.2437(8)$ & $0.418(2)$ & 18 & 1.0 & $0.020(5)$ \\
$\mathrm{C} 2$ & $0.3217(8)$ & $0.2094(8)$ & $0.286(2)$ & 18 & 1.0 & $0.024(5)$ \\
$\mathrm{C} 3$ & $0.3404(7)$ & $0.2229(9)$ & $0.087(2)$ & 18 & 1.0 & $0.006(5)$ \\
$\mathrm{C} 4$ & $0.3471(7)$ & $0.1815(7)$ & $-0.025(2)$ & 18 & 1.0 & $0.004(5)$ \\
$\mathrm{H}$ & $0.375(1)$ & $0.196(1)$ & $-0.183(4)$ & 18 & 1.0 & $0.002(7)$ \\
$\mathrm{D}_{2}(1)$ & $0.2006(8)$ & $-0.0108(9)$ & $0.062(2)$ & 18 & $1.76(4)$ & $\mathrm{a}$ \\
$\mathrm{D}_{2}(2)$ & $0.4903(7)$ & $0.1191(7)$ & $-0.010(3)$ & 18 & $1.89(6)$ & $0.18(1)$ \\
$\mathrm{D}_{2}(3)$ & $0.748(1)$ & $0.241(1)$ & $0.129(3)$ & 18 & $1.87(5)$ & $0.22(1)$ \\
$\mathrm{D}_{2}(4)$ & $0.638(7)$ & $0.265(5)$ & $-0.06(2)$ & 18 & $0.19(2)$ & $0.12(7)$ \\
\hline
\end{tabular}

a) The anisotropic thermal factor for $D_{2}(1)$ :

\begin{tabular}{|c|c|c|c|c|c|}
\hline $\mathrm{U}_{11}$ & $\mathrm{U}_{22}$ & $\mathrm{U}_{33}$ & $\mathrm{U}_{12}$ & $\mathrm{U}_{13}$ & $\mathrm{U}_{23}$ \\
$0.19(2)$ & $0.20(2)$ & $0.10(1)$ & $0.10(2)$ & $0.02(1)$ & $-0.01(1)$ \\
\hline
\end{tabular}


Table S8. Rietveld refinement results for $4.2 \mathrm{D}_{2}: Z n . a=b=25.882(2) \AA, c=6.8614(4)$

$\AA$. Estimated standard deviations from the Rietveld analysis are given in parentheses.

\begin{tabular}{|c|c|c|c|c|c|c|}
\hline & $\mathrm{x}$ & $\mathrm{y}$ & $\mathrm{z}$ & Multiplicity & Occupancy & $\mathrm{U}_{\text {iso }}\left(\AA^{2}\right)$ \\
\hline $\mathrm{Zn}$ & $0.3841(6)$ & $0.3567(6)$ & $0.132(2)$ & 18 & 1.0 & $0.017(4)$ \\
$\mathrm{O} 1$ & $0.3213(5)$ & $0.2930(6)$ & $0.363(2)$ & 18 & 1.0 & $0.009(3)$ \\
$\mathrm{O} 2$ & $0.3020(6)$ & $0.2230(5)$ & $0.609(2)$ & 18 & 1.0 & $0.012(4)$ \\
$\mathrm{O} 3$ & $0.3551(6)$ & $0.2759(6)$ & $-0.009(2)$ & 18 & 1.0 & $0.018(4)$ \\
$\mathrm{C} 1$ & $0.3148(6)$ & $0.2417(5)$ & $0.429(2)$ & 18 & 1.0 & $0.024(3)$ \\
$\mathrm{C} 2$ & $0.3222(6)$ & $0.2047(7)$ & $0.292(2)$ & 18 & 1.0 & $0.040(4)$ \\
$\mathrm{C} 3$ & $0.3426(5)$ & $0.2173(6)$ & $0.084(1)$ & 18 & 1.0 & $0.016(3)$ \\
$\mathrm{C} 4$ & $0.3516(5)$ & $0.1817(6)$ & $-0.028(1)$ & 18 & 1.0 & $0.009(3)$ \\
$\mathrm{H}$ & $0.368(1)$ & $0.203(1)$ & $-0.169(3)$ & 18 & 1.0 & $0.032(7)$ \\
$\mathrm{D}_{2}(1)$ & $0.2002(4)$ & $-0.0104(5)$ & $0.034(1)$ & 18 & $1.76(3)$ & $\mathrm{a}$ \\
$\mathrm{D}_{2}(2)$ & $0.4924(6)$ & $0.1174(7)$ & $-0.017(3)$ & 18 & $2.13(5)$ & $0.413(9)$ \\
$\mathrm{D}_{2}(3)$ & $0.7489(4)$ & $0.2359(4)$ & $0.169(1)$ & 18 & $2.07(3)$ & $0.168(4)$ \\
$\mathrm{D}_{2}(4)$ & $0.6214(9)$ & $0.2484(6)$ & $0.041(1)$ & 18 & $2.01(2)$ & $0.43(1)$ \\
\hline
\end{tabular}

a) The anisotropic thermal factor for $D_{2}(1)$ :

\begin{tabular}{|c|c|c|c|c|c|}
\hline $\mathrm{U}_{11}$ & $\mathrm{U}_{22}$ & $\mathrm{U}_{33}$ & $\mathrm{U}_{12}$ & $\mathrm{U}_{13}$ & $\mathrm{U}_{23}$ \\
$0.009(5)$ & $0.178(9)$ & $0.21(1)$ & $0.059(7)$ & $-0.029(7)$ & $-0.032(8)$ \\
\hline
\end{tabular}


Table S9. Adsorption data of hydrogen at $77 \mathrm{~K}$ and $87 \mathrm{~K}$ in MOF-74

\begin{tabular}{|rr|rr|}
\hline \multicolumn{2}{|c|}{ Adsorption at 77K } & \multicolumn{2}{c|}{ Adsorption at 87K } \\
\hline Pressure (bar) & $\mathrm{H}_{2}$ uptake $\mathrm{wt} \%$ & Pressure $(\mathrm{bar})$ & $\mathrm{H}_{2}$ uptake $(\mathrm{wt} \%)$ \\
\hline 0.001067 & 0.081587 & 0.013732 & 0.199055 \\
0.002266 & 0.162966 & 0.025864 & 0.319707 \\
0.003466 & 0.234386 & 0.033597 & 0.381382 \\
0.004933 & 0.302733 & 0.046529 & 0.464737 \\
0.006666 & 0.372873 & 0.065193 & 0.554824 \\
0.008932 & 0.442359 & 0.09839 & 0.666882 \\
0.011865 & 0.514474 & 0.165183 & 0.806765 \\
0.015598 & 0.582977 & 0.258241 & 0.927983 \\
0.021998 & 0.668086 & 0.36983 & 1.028548 \\
0.03613 & 0.782497 & 0.490084 & 1.111756 \\
0.06266 & 0.900745 & 0.628737 & 1.190174 \\
0.094657 & 0.989041 & 0.844716 & 1.289998 \\
0.136653 & 1.071726 & 1.139886 & 1.400219 \\
0.178915 & 1.137105 & 1.463987 & 1.499628 \\
0.231044 & 1.187456 & 1.794054 & 1.584954 \\
0.258241 & 1.235567 & 2.16535 & 1.66767 \\
0.295837 & 1.275399 & 2.509582 & 1.735357 \\
0.339433 & 1.294956 & & \\
0.426891 & 1.368252 & & \\
0.549145 & 1.454244 & & \\
0.708196 & 1.547157 & & \\
0.855114 & 1.619169 & & \\
1.148152 & 1.735238 & & \\
1.495184 & 1.842174 & & \\
1.902443 & 1.941349 & & \\
2.373996 & 2.033368 & & \\
2.948605 & 2.127143 & & \\
3.701363 & 2.219518 & & \\
4.710596 & 2.3232 & & \\
5.923808 & 2.427725 & & \\
7.651635 & 2.542244 & & \\
9.380795 & 2.611613 & & \\
12.11785 & 2.662067 & & \\
14.94824 & 2.70832 & & \\
17.9516 & 2.751414 & & \\
21.25927 & 2.781282 & & \\
24.85225 & 2.751042 & & \\
27.93727 & 2.737531 & & \\
31.20095 & 2.73374 & & \\
& & & \\
\hline
\end{tabular}


Table S10. Isosteric heats of adsorption at different coverage (i. e. for various $\mathrm{H}_{2}$ uptake in wt.\%)

\begin{tabular}{|r|r|}
\hline $\mathbf{H}_{\mathbf{2}}(\mathbf{w t} \%)$ & $\mathbf{Q} \mathbf{( k J / m o l})$ \\
\hline 0.2 & 8.805 \\
0.3 & 8.860 \\
0.4 & 8.814 \\
0.5 & 8.741 \\
0.6 & 8.620 \\
0.7 & 8.411 \\
0.8 & 7.854 \\
0.9 & 7.414 \\
1 & 6.792 \\
1.1 & 6.266 \\
1.2 & 5.532 \\
1.3 & 5.120 \\
1.4 & 4.916 \\
1.5 & 4.725 \\
1.6 & 4.586 \\
\hline
\end{tabular}




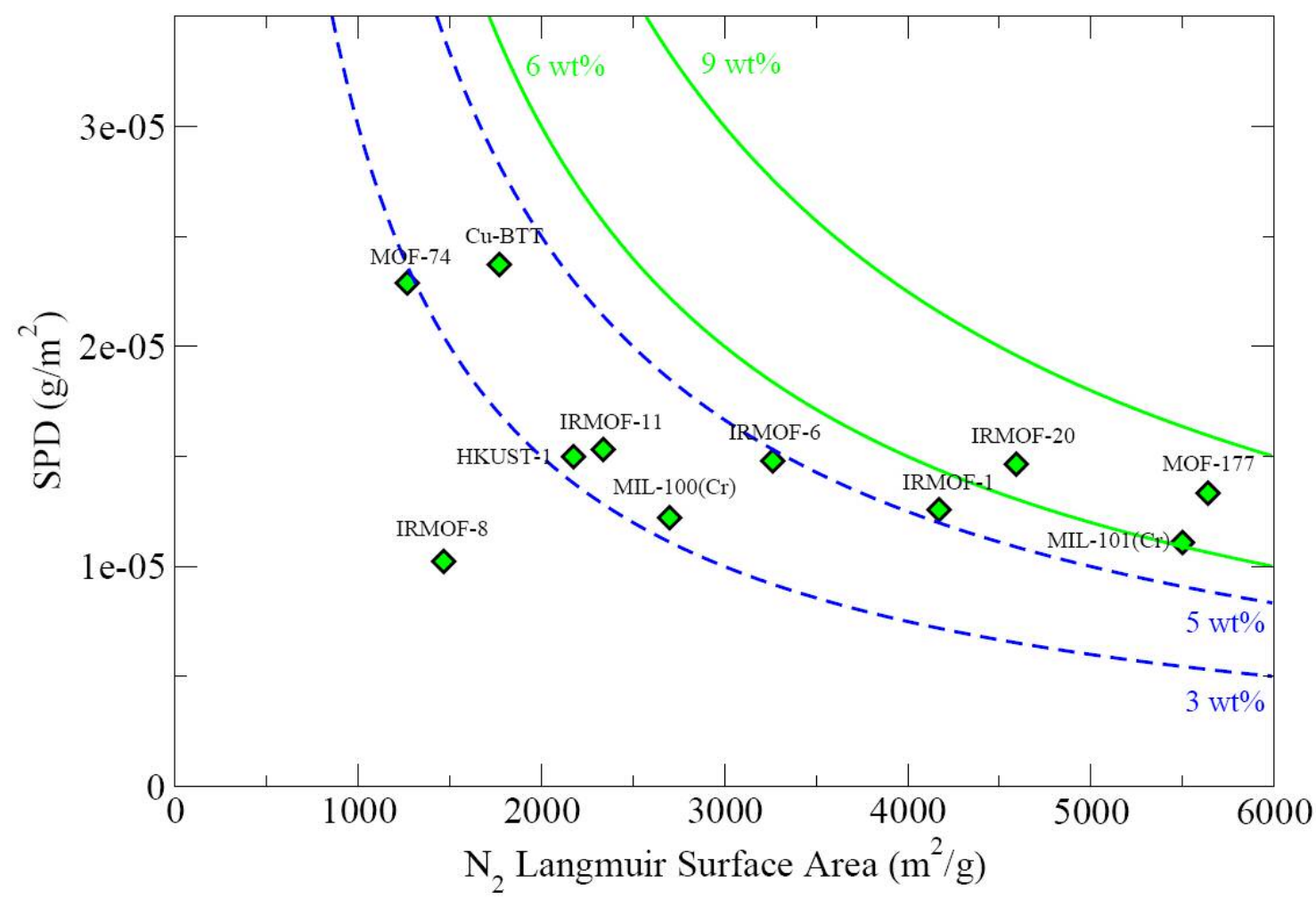

Fig. S1. If we define $S$ as the $\mathrm{N}_{2}$ Langmuir surface area in the definition of $S P D$, we can calculate the $S P D$ based on the Langmuir surface area. The calculated $S P D$ as a function of $S$ is shown here. Because some MOFs do not have information reported for both BET and Langmuir surface, these MOFs reported in Fig.4 of the manuscript are not shown in the current figure. We have also added the results of MIL-100(Cr) and MIL-101(Cr) ${ }^{5}$ in the current figure.

\footnotetext{
${ }^{5}$ M. Latroche et al. Angew. Chem. Int. Ed 45, 8227-8231 (2006).
} 


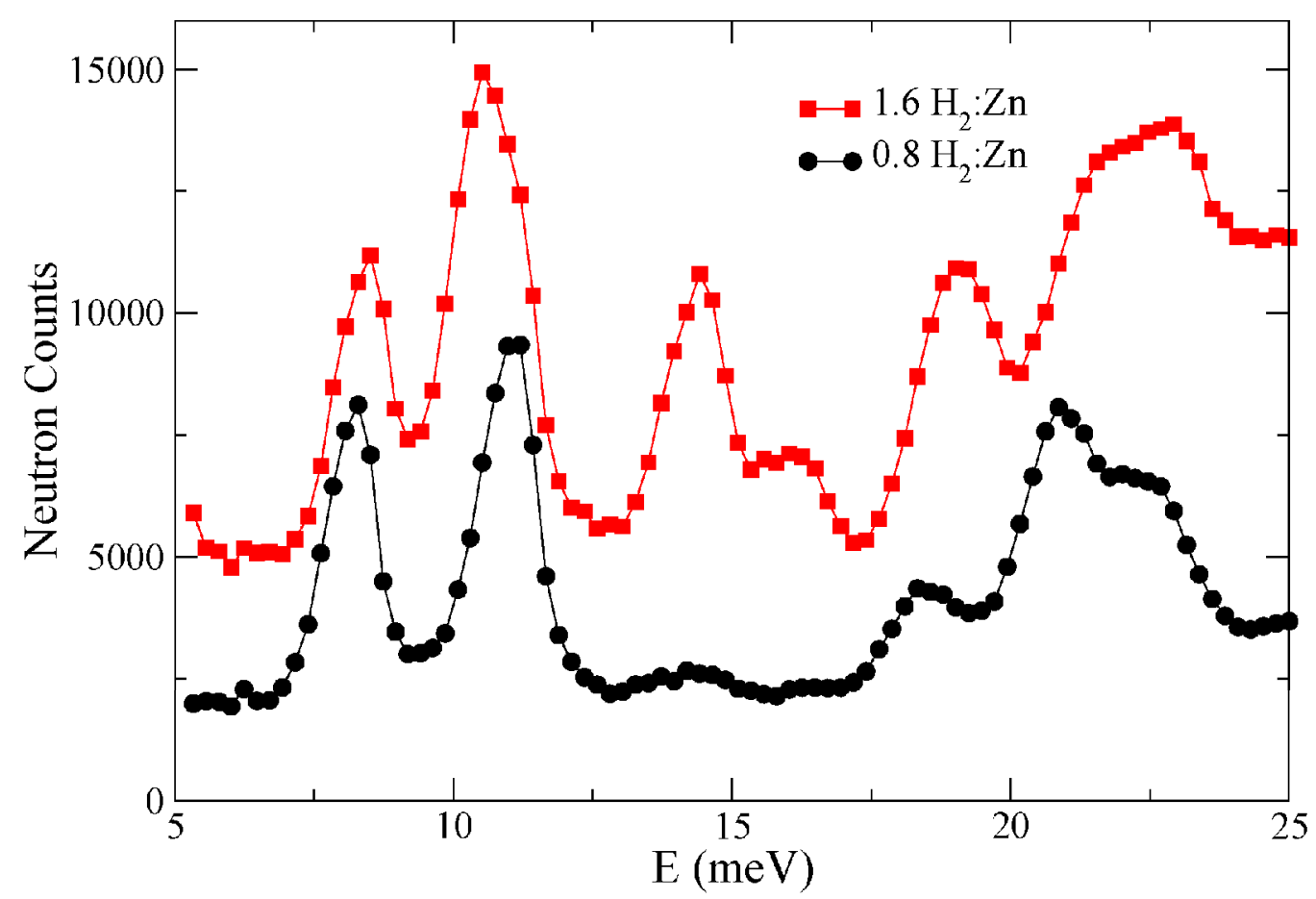

Fig. S2. The inelastic neutron scattering spectra of adsorbed $\mathrm{H}_{2}$ at different gas loadings are shown. All measurements were performed at $4 \mathrm{~K}$. Error bars are smaller than the symbols. 


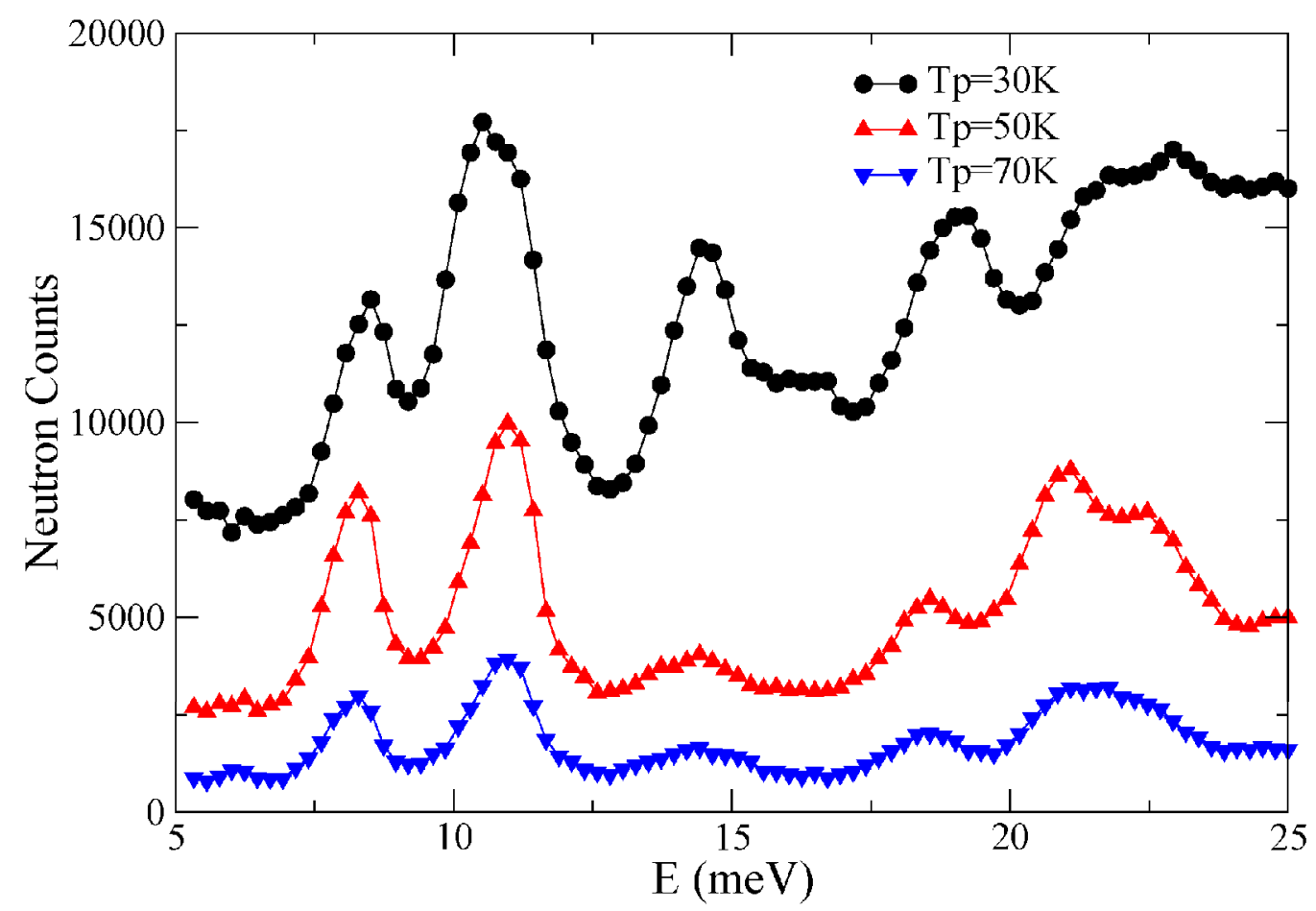

Fig. S3. The inelastic neutron scattering spectra of adsorbed $\mathrm{H}_{2}$ as a function of degassing temperature, $T_{p} .2 .8 \mathrm{H}_{2}: \mathrm{Zn}$ was first loaded into the sample and the sample was cooled down to $4 \mathrm{~K}$ before being warmed up to the degassing temperature, $T_{p}$, where $\mathrm{H}_{2}$ gas was pumped out using a turbo-pump for about 30 minutes. After degassing $\mathrm{H}_{2}$ at $T_{p}$, the sample was cooled down to $4 \mathrm{~K}$ for the neutron scattering measurements. $T_{p}$ was sequentially raised from $30 \mathrm{~K}$ to $50 \mathrm{~K}$ and then $70 \mathrm{~K}$. Error bars are smaller than the symbols. 


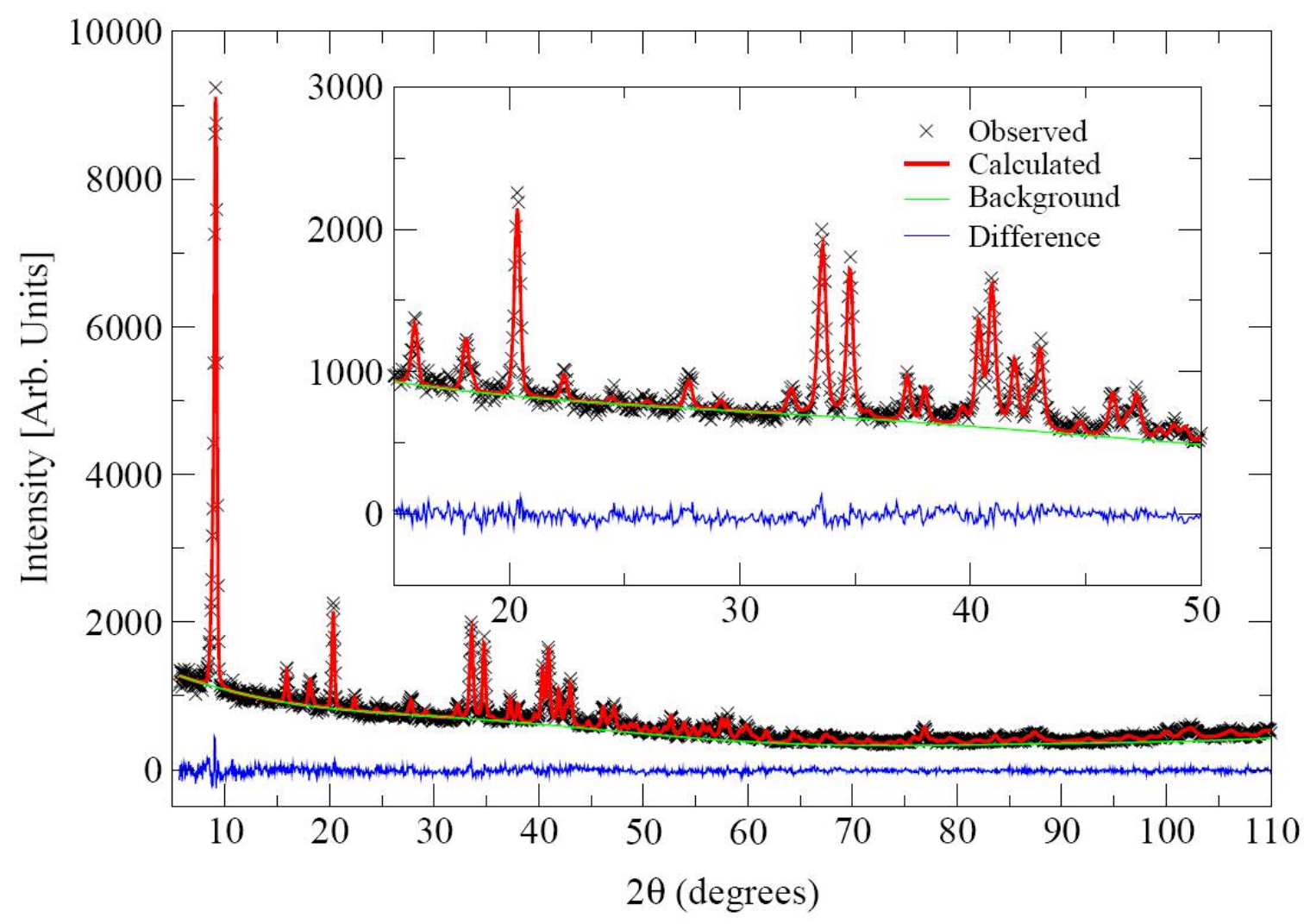

Fig. S4. Rietveld refinement and neutron powder diffraction data for a loading at 3.0 $\mathrm{D}_{2}: \mathrm{Zn}$. Cross, red line, green line, and blue line represent the experimental diffraction pattern, calculated pattern, calculated background, and the difference between experiment and calculated patterns. Data were collected using neutrons of wavelength $2.0787 \AA$. 